\title{
Research on Camouflage Assessment Contrast of Different Pattern Paintings under Visional Condition
}

\author{
Jia Qi ${ }^{a}$, Lv Xu Liang ${ }^{b}$, Xu Wei Dong $^{c}{ }^{\text {* }}$, Hu Jiang Hua ${ }^{d}$ and Liu Jun ${ }^{\mathrm{e}}$ \\ College of Field Engineering, PLA University of Science and Technology, Nanjing, 210007, China

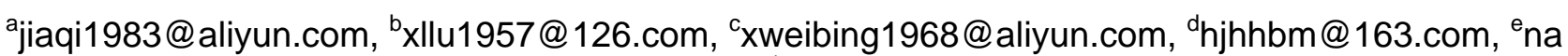 \\ njnliujun@163.com, ${ }^{*}$ corresponding author
}

\begin{abstract}
Keywords: Camouflage Evaluation, Pattern Painting, Visional Condition, Identification Probability, Detection Probability
\end{abstract}

\begin{abstract}
The camouflage effect evaluation of camouflage pattern painting is based on the values of detection and identification probabilities. A number of observers are organized to observe the targets in field traditionally, and then the observation results are statistical analyzed to quantify camouflage effect. However, this method is time and money consuming, also relatively poor reproducibility. With the development of surveillance technology, the traditional form camouflage technologies face serious challenges, and then digital pattern painting emerged. And it is a problem that how to quantitatively evaluate camouflage effect of traditional and digital pattern paintings. In this paper, the field experiments are organized in woodland and desert backgrounds to evaluate camouflage effect of the traditional and digital pattern painting in eastern suburbs of Nanjing city, based on the model of detection and identification probabilities. The results show that the calculation results based on the model is consistent with actual experimental results, and the camouflage effect of digital pattern painting is better than that of traditional pattern painting. The calculation model in this paper is a useful method in quantitative camouflage effect evaluation of camouflage pattern painting.
\end{abstract}

\section{Introduction}

In the process of camouflage pattern painting design, the designed pattern paintings should be assessed and evaluated firstly and selected according to the camouflage effects [1]. Moreover, the camouflage evaluation should be quantified. Therefore, it is particularly important that how to quantitatively evaluate the camouflage effect of the pattern painting. The camouflage effect evaluation is based on the degree of blending effect between the object and surrounding background in term of shape, size, color and spatial distribution [2, 3]. Traditionally, A number of observers are organized to observe the targets in field at a certain distance all along, and then the observation results are statistical analyze to quantify the camouflage effect in detection and identification probabilities. The traditional method to measure detection and identification probabilities is accurate. However, this method is time and money consuming, also relatively poor reproducibility $[4,5]$. Therefore, the method only is used in camouflage effect evaluation of a handful of important objectives.

When some relative conditions changed, it is necessary to reorganized the experiment according to the traditionally method. Limited by time and money, the field experiments is only a small amount, which cannot objectively reflect the adaptability of the entire region. Therefore, it is very necessary that the alternative methods should be researched which effectively adapt to a variety of target camouflage battlefield environments. With the development of surveillance technology, the traditional form of camouflage face serious challenges, and then digital pattern painting emerged. And there are problems that how to quantitatively evaluate the camouflage effect of traditional and digital pattern paintings, and how to construct the relation between camouflage effect and detection and identification probabilities. 


\section{Detection Probability under Visual Condition}

Detection probability is the probability of the target is detected, in the form of percentage. $0 \%$ is that the target will not be detected to a certainty. $100 \%$ is that the target will be detected all to nothing. The detection probability greater, the probability of target is more likely found, and the easier the target detected, and the more dangerous to the target. Therefore, the value of detection probability should be reduced.

For camouflage pattern painting, the primary reconnaissance manner to deal with is the visual reconnaissance. Therefore, the detection probability under visual condition is researched here.

According to the camouflage theory, the typical target detection probability can be calculated under visual observation conditions by the following formula as follow

$$
P_{d}=\frac{1}{\sqrt{2 \pi}} \int_{-\infty}^{\left(\frac{K}{\varepsilon}-1\right) / \sigma} \exp \left(-\frac{x^{2}}{2}\right) d x
$$

Where, $K$ is the target contrast observed by human eye, $\varepsilon$ is the threshold of luminance contrast, $\sigma=0.39$. In the monotonous background, brightness and contrast thresholds are as follow

$$
\varepsilon= \begin{cases}0.02 & \theta>30^{\prime} \\ 0.325 \theta^{-0.819} & \theta \leq 30^{\prime}\end{cases}
$$

As for the luminance contrast threshold mottled background is multiplied by the coefficient 2.5, which are as

$$
\varepsilon= \begin{cases}0.05 & \theta>30^{\prime} \\ 0.8125 \theta^{-0.819} & \theta \leq 30^{\prime}\end{cases}
$$

Where, $\theta$ is the target opening angle of the human eye, which is as follow

$$
\theta=\Gamma \frac{d}{l}
$$

Where, $\Gamma$ is the magnification of the optical observation equipment, $d$ is the size of target, $l$ is the reconnaissance distance.

When the viewer observes the target, due to atmospheric attenuation, brightness contrast target and background will be attenuated, called brightness contrast, the relationship between the brightness contrast $K^{\prime}$ and target and background brightness contrast $K$ is as follows

$$
K^{\prime}=\frac{K}{1-\frac{L_{H}}{L_{B}}\left(1-\tau^{-L}\right)}
$$

Where, $\tau$ is the atmosphere transparency coefficient, when the weather is generally sunny $\tau=0.822$, which is equivalent to weather visibility $s=20 \mathrm{~km}$, the sky brightness horizon $L_{H}$, the brightness of the background $L_{B}$.

\section{Identification Probability under Visual Condition}

In the instant of detecting the target, the target should be resolved which determine whether it is the goal looking for or not, that is identification. Detection and identification are two closely linked inseparable events. Under different circumstances, the identification requirements are different, generally divided into four degrees, such as doubt discovered, confirmed the target, identify the target, and describe the target. In different searching equipment, the identification modes are different.

Identification probability of camouflage target is the probability of target is be identified and recognized in a given distance. The smaller of identification probability, represents the target camouflage is better.

Under visual observation conditions, the typical target identification probability can be expressed by the following equation. When the detection probability $P_{d}<90 \%$, the target cannot be identified and recognized, and the identification probability $P_{r}=0$. When the detection probability $P_{d} \geq 90 \%$, 


$$
\varepsilon_{0}=\frac{K^{\prime}}{1.5}
$$

The detail of the diagonal dimension of the target $\theta_{0}$ can be obtained, when the equation (6) join the equation (4). Then, the target shape smallest transverse dimension of the details contained in the distinguished number is as

$$
N_{r}=\frac{\theta}{\theta_{0}}
$$

Then, the target identification probability is as follow

$$
P_{r}= \begin{cases}0 & N_{r}<2 \\ 1-e^{-\left[\frac{N_{r}}{2}-1\right]^{2}} & N_{r} \geq 2\end{cases}
$$

\section{Traditional And Digital Camouflage Pattern Painting}

The camouflage pattern painting is that coatings, dyes and other materials are used to change the spectral reflectance characteristics of target, screening, and surface background. Effective camouflage renders a target indistinguishable from irrelevant background objects [6]. The animal's colors in nature have two different camouflage methods: background pattern matching $[7,8]$ and disruptive coloration $[9,10]$.

The camouflage pattern painting is the most basic camouflage technique. It is widely used in many targets using camouflage paint. And it can be done fast, and be effective fast. Many other camouflage measures can be done on the basis of camouflage pattern painting. However, the camouflage effect of pattern painting directly applied to the target is limited. Generally, the color difference of target and background is decreased, and the significant degree is reduced, and the target shape is changed.

In order to prevent the space mixing phenomenon of spots in various colors, ensure multi-color camouflage deformation, camouflage spot size must be visible at a predetermined viewing distance.

Spot visible size mainly depends on the observation distance, the brightness difference between spots, and the degree of atmosphere transparency. In general transparent atmospheric conditions ( $\tau=0.8 \sim 0.9$ ), spots of visible size limit can be approximate computed by the following formula.

When observing with naked eye and the brightness contrast between spots satisfies $K \geq 0.4$, the equation is as

$$
d \geq \frac{(2.5 \sim 3) D}{3438}
$$

When the brightness contrast between spots satisfies $0.2<K<0.4$, the spot size in visible should be increased as

$$
d \geq \frac{(3.5 \sim 4) D}{3438}
$$

Where, $d$ is the size of spot. $D$ is the observation distance which only considering the case of ground and close air observation. Because of their small size, such as artillery, tanks, vehicles and other targets, they can be detected and found in closer viewing distance.

In order to prevent the shapes and sizes of camouflage spots are stereotyped, the various sizes camouflage spots should be taken as far as possible on the basis of that they are visible.

Digital camouflage pattern painting, also known as numeral camouflage pattern painting, is a type of camouflage pattern painting that camouflage multicolor block unit is generated from the digital imaging and processing technology. It is a new camouflage pattern painting designed technology. It is based on the biological characteristics of the human visual system, based on the pixel expression of background image feature. Base on the space blending theory, according to computer-aided design or computer-generated design, a new camouflage pattern painting having dual texture of macro and micro pattern constructed by basic units is generated. 
Morphological characteristics of space blending effect analysis showed that the square is the most stable form and the color can be fully set, easily form space blending, and also easily coating operation, which is consistent with CCD detector element array. Therefore, the square unit is using in digital camouflage pattern painting. The size of the basic unit of digital camouflage pattern painting is smaller, the more sophisticated, the degree of background simulation is higher. When the basic unit is too small, camouflage effect under conditions of photographic reconnaissance satellite targets will not be significantly improved. And the pattern design and painting construction are more difficulty. Therefore, the size of the basic unit of digital camouflage pattern painting needs to consider the spatial resolution of reconnaissance equipment dealing with, human eye visual acuity, pattern design, painting operability and other factors. Through the establishment relation model between detection probability of digital pattern painting and basic unit size under photographic satellite reconnaissance, and the physical relationship between the basic unit of human retinal image size and target size, combining with the tactical requirements against photographic reconnaissance satellite, taking into account painting operability, the basic unit size is determined.

When the length of basic spot unit is $0.1 \mathrm{~m}$, it is difficult to distinguish a single basic unit under the condition of photographic reconnaissance satellite. The transition between various units is smooth, and spot color blending effect is generating. To minimize the painting difficulty on the basis of not affect camouflage effect, the basic unit of $10 \mathrm{~cm} \times 10 \mathrm{~cm}$ is more appropriate. At the same time, in order to enhance camouflage effect by optical illusion, blend to surrounding background, a small amount of modification spots can be deployed, in the basic unit size of $5 \mathrm{~cm} \times 5 \mathrm{~cm}$.

\section{Experiment}

An example is selected to calculated and experimented. The experiment is done in sunny weather in eastern suburb of Nanjing city. The atmosphere transparency coefficient $\tau=0.822$, which is equivalent to weather visibility $s=20 \mathrm{~km}$. The target transverse dimension is $2.8 \mathrm{~m}$. The observing distance $l=1.5 \mathrm{~km}$. The telescope magnification is $10 \times$. The original contrast between target and background is 0.4. The experiments are done in two backgrounds, woodland and desert. Under normal circumstances, when the sunny weather, woodland type background $L_{H} / L_{B}=5$, desert type background $L_{H} / L_{B}=1.4$. The targets are painted in the forms of traditional and digital pattern painting. The distinguished size of traditional pattern painting is $0.6 \mathrm{~m}$, and the basic unit size of digital pattern painting is $0.1 \mathrm{~m}$. These two forms camouflage pattern paintings have the same color scheme and in the same backgrounds.

In order to verify the correctness of the model calculation, 60 observers are organized to observe the target camouflage effect in woodland and desert backgrounds. Then the observation results are statistical analyzed. The detection probabilities of traditional and digital camouflage pattern painting in woodland and desert background are separately actually measured and calculated, as shown in Table.1. The detection and identification probabilities of traditional and digital camouflage pattern painting in woodland and desert background are separately calculated, as shown in Table.2.

Table.1 The measured and calculated detection probabilities of traditional and digital camouflage pattern painting in woodland and desert background

\begin{tabular}{|c|c|c|c|c|}
\hline \multirow{2}{*}{ Detection probability } & \multicolumn{2}{|c|}{ Woodland } & \multicolumn{2}{c|}{ Desert } \\
\cline { 2 - 5 } & Calculated & Measured & Calculated & Measured \\
\hline Traditional camouflage & 1.0 & 0.9830 & 1.0 & 1.0 \\
\hline Digital camouflage & 0.3950 & 0.4167 & 1.0 & 0.9667 \\
\hline
\end{tabular}

Table.2 The calculated detection and identification probabilities of traditional and digital camouflage pattern painting in woodland and desert background

\begin{tabular}{|c|c|c|c|c|}
\hline \multirow{2}{*}{ Probability } & \multicolumn{2}{|c|}{ Woodland } & \multicolumn{2}{c|}{ Desert } \\
\cline { 2 - 5 } & Detection & Identification & Detection & Identification \\
\hline Traditional camouflage & 1.0 & 0 & 1.0 & 0.0085 \\
\hline Digital camouflage & 0.3950 & 0 & 1.0 & 0 \\
\hline
\end{tabular}




\section{Summary}

The results showed that the measured and calculated detection probabilities are consistent. The calculation error is less than $4 \%$. Therefore, the model can be used to quantitatively evaluate camouflage effect of pattern painting. Meanwhile, the results showed that the camouflage effect of digital pattern painting is better than that of traditional pattern painting in the same background. In woodland, the detection probability of traditional camouflage is $100 \%$, and that of digital camouflage is only $39.5 \%$. In desert, the detection probabilities of traditional and digital camouflage are same, which are $100 \%$, which mean that they are will be detected. However, the identification probability of digital camouflage is $0 \%$, and the traditional probability of digital camouflage is $0.85 \%$, which is small higher than that of digital camouflage. However, in the processing of calculation, the using smallest unit size of digital camouflage as the size of target has the appropriate prerequisite, which is that digital camouflage pattern painting must be carefully designed to be able to form a dual camouflage pattern texture pattern macro and micro pattern, which is difficult.

The process of detection, recognition, and identification of camouflaged target is very complex. And there are many factors, which are difficult to quantify and affect the process, such as the experience of observer, physiological and psychological status, degree of effort, etc. Also the camouflage target state affects the process, such as the distribution of the spots, the degree of amorphous spots, the degree of target segmentation distorted. These factors are not discussed in the above analysis. Thus, the above analysis is according to the camouflage principle, and simplified which can basically reflect current research situation. In short, the calculation model in this paper is a useful method in quantitative camouflage effect evaluation of camouflage pattern painting, which comprises traditional and digital camouflage pattern painting.

\section{References}

[1] W. D. Xu, X. L. Lv, B. Chen and S. Q. Xue, A model based on texture analysis for the performance evaluation of camouflage screen equipment, Acta Armamentarii. 23 (2002) 329-331.

[2] W. Lin, Y. H. Chena, H. S. Gao, L. Lin and J. J. Wang, A method of camouflage evaluation based on texture analysis model of Gabor wavelet, Acta Armamentarii. 28 (2007) 1191-1194.

[3] Y. J. Wang, X. L. Lv and J. H. Hu, Gray correlation analysis method for scheme selection decision of camouflage screen design, Systems Engineering and Electronics. 31 (2009) 618-620.

[4] Q. Jia, X. L. Lv, C. Wu and H. C. Tang, Evaluation of camouflage effectiveness using human visual attention mechanisms, Journal of Applied Sciences. 29 (2011) 294-298.

[5] Q. Jia, X. L. Lv, Z. Y. Zeng and W. D. Xu, Application of descriptor in valuation of target camouflage effectiveness, Journal of Applied Sciences. 29 (2011) 483-486.

[6] I. C. Cuthill, M. Stevens, J. Sheppard, T. Maddocks, C. A. Parraga and T. S. Troscianko, Disruptive coloration and background pattern matching, Nat. 434 (2005) 72-74.

[7] J. A. Endler, Progressive background in moths, and a quantitative measure of crypsis, Biol. J. Linn. Soc. 22 (1984) 187-231.

[8] J. A. Endler, An overview of the relationships between mimicry and crypsis, Biol. J. Linn. Soc. 16 (1981) 25-31.

[9] G. H. Thayer, Concealing Coloration in the Animal Kingdom; An Exposition of the Laws of Disguise through Color and Pattern; Being a Summary of Abbott H. Thayer's Discoveries, 1909, New York, Macmillan.

[10] S. Merilaita, Crypsis through disruptive coloration in an isopod, Proc. R. Soc. Lond. B265, 1998, 1059-1064. 